\title{
DETECTION OF HYPERMUCOVISCOUS KLEBSIELLA PNEUMONIAE AND PHENOTYPIC COMPARISON OF THEIR VIRULENCE FACTORS WITH CLASSICAL STRAINS AMONG PATIENTS VISITING TERTIARY CARE HOSPITAL
}

Tika Bahadur Thapa ${ }^{1,2}$ Sujina Maharjan ${ }^{1,3}$ Nisha Giri ${ }^{1}$ Manisha Sapkota ${ }^{1}$ Ojaswee Shrestha ${ }^{1,2}$ Puspa Raj Khanal ${ }^{1,2}$ Govardhan joshi ${ }^{1,4}$

\author{
${ }^{1}$ Department of Laboratory Medicine, Manmohan Memorial Institute of Health Sciences, \\ Kathmandu, Nepal \\ ${ }^{2}$ Department of Pathology, Sumeru Hospital Pvt Ltd, Dhapakhel, Lalitpur, Nepal \\ ${ }^{3}$ Department of Pathology, Manmohan Memorial Teaching Hospital, Kathmandu, Nepal \\ ${ }^{4}$ Department of Pathology, Global Hospital and Education Foundation, Gwarko, Lalitpur, \\ Nepal
}

\section{Corresponding author}

Mr. Govardhan Joshi

Email: govardhan joshi2001@yahoo.com

Mobile No: $+977-9849669766$

\section{Abstract}

Background: Klebsiella pneumoniae, which is commonly recognized in the laboratory, are termed as classical K. pneumoniae (cKP). A new strain of Klebsiella pneumoniae named hypermucoviscous K. pneumoniae (hmvKP) emerging with a distinctive feature than classical strains. In many recent studies, infections due to hypermucoviscous strains have been increasing with significant mortality and morbidity. Our study aimed to determine the prevalence of hypermucoviscous Klebsiella pneumoniae in our hospital and phenotypic comparison of their virulence with the classical strains.

Materials and Methods: This laboratory-based cross-sectional study done from February to July 2018 in the Department of Microbiology, Manmohan Memorial Teaching Hospital, Kathmandu, Nepal. One hundred and five clinical non-duplicative isolates of Klebsiella pneumoniae proceeded for the study. Hypermucoviscosity of isolated Klebsiella pneumoniae were tested by standard 
String test. Antibiotic susceptibility was determined by the Kirby-Bauer disk diffusion method according to Clinical and Laboratory Standards Institute (CLSI) guidelines. Further, $\beta$-lactamases (ESBL, MBL, and KPC) were detected by the phenotypic combination disk test method. Serum resistance and biofilm production were determined to assess their virulence.

Results: A total of one hundred five non-repetitive pure and microbiologically identified Klebsiella pneumoniae were isolated from various clinical samples collected during the study period. Among the isolated Klebsiella pneumoniae, 29 (27.6\%) were hypermucoviscous K. pneumoniae (hmvKP), and 76(72.4\%) were classical K. pneumoniae (cKP) determined by string test. The majority of Klebsiella pneumoniae were resistant to ceftazidime $(80 \%)$ and cefotaxime $(78 \%)$, whereas $46.7 \%$ of isolates were resistant to both imipenem and meropenem. Various virulence factors, namely beta-lactamase production, biofilm, and serum resistance, were compared among classical and hypermucoviscos strains, and the hypermucoviscous strains revealed significantly higher expression of virulence than classical strains.

Conclusion: The phenotypic comparison of virulence factors and Beta-lactamase production was found significantly higher among the hypermucoviscous strains. The prevalence of these hypermucoviscous strains in hospital settings may increase the risk of morbidity and mortality. Therefore, timely diagnosis and appropriate treatment strategies to limit these infections are crucial.

Keywords: Hypermucoviscosity, Klebsiella pneumoniae, Multi-Drug Resistance, Biofilm, Serum resistance, Nepal

\section{Introduction}

Klebsiella pneumoniae was first isolated by Carl Friedlander in 1882 and initially known as Friedlander's bacterium (1). They are Gram-negative, non-motile, encapsulated bacterium of family Enterobacteriaceae that resides in the environments $(2,3) . K$. pneumoniae can readily colonize in human mucosal surfaces, including the gastrointestinal (Gl) tract and oropharynx, from where their colonization begins (2-4). They are found to be responsible for many communityonset and nosocomial infections. Typically, classical $K$. pneumoniae strains cause severe 
infections like pneumonia, bacteremia, or meningitis, including in immunocompromised peoples such as diabetes or malignancies (5-7). However, since the 1980s, a new strain of $K$. pneumoniae was found to be causing serious infections in healthy individuals and these strains are considered hypermucoviscous (hypervirulent) as compared to classical $K$. pneumoniae strains due to its ability to infect both immunocompetent and immunocompromised populations, and they have increased tendency of infections to be invasive; i.e., they can establish metastatic infections (8-10).

Over the past few decades, K. pneumoniae are increasing to resistant with the majority of antibiotics by applying different resistance mechanisms. Consequently making difficulty in treating uncomplicated infections like urinary tract infections, and serious infections like bacteremia's, pneumonia, and becoming the life-threatening $(11,12)$. Majorly two types of resistance mechanisms have been commonly observed in $K$. pneumoniae. First is the expression of extended-spectrum $\beta$-lactamases (ESBLs) (13), which render bacteria resistant to penicillin, cephalosporin, and monobactam whereas the second common mechanism of resistance is the expression of carbapenemase by $K$. pneumoniae, which makes bacteria resistant to almost all available $\beta$-lactam antibiotics, including the carbapenem drugs (14). Increasing incidence of ESBL and carbapenemase-producing $K$. pneumoniae is a global concern, and this scenario is exacerbated when they develop biofilm production by causing increase resistance to multiple antimicrobial agents (13).

There is an increasing prevalence of hypermucoviscous $K$. pneumoniae among the Asian countries, and to this date, there was no study documented on infection of hypermucoviscous strains in Nepal. Therefore, we aimed to study the prevalence of hypermucoviscous $K$. pneumoniae in our hospital settings and phenotypically compare their virulence with the classical strains. 


\section{Material and methods}

The laboratory-based cross-sectional study was done from February to July 2018 in the department of microbiology, Manmohan Memorial Teaching Hospital, Swoyambhu, Kathmandu, Nepal. All the clinical samples received in the microbiology department for culture were included in the study.

\section{Bacterial isolation and identification;}

Clinical samples collected in the microbiology department were inoculated on appropriate culture media. Urine samples were inoculated on Cystine-lactose-electrolyte-deficient (CLED) agar (HiMedia, India), Sputum samples were inoculated on Chocolate, Blood, and Mac Conkey agar (HiMedia, India). Similarly, wound swabs were inoculated on Blood, and Mac Conkey agar and Blood samples were first inoculated on Brain Heart Infusion broth. They then sub-cultured on Blood and Mac Conkey agar. Hundred and five isolates of $K$. pneumoniae were isolated from various clinical specimens during the period. All the $K$. pneumoniae isolates were identified by standard microbiological tests. The protocol conducted in the study complies with the ethical guidelines adopted by the Institutional Review Committee (IRC) of Manmohan Memorial Institute of Health Sciences, Soalteemode, Kathmandu, Nepal (IRC no.-228).

\section{Antimicrobial Susceptibility Testing;}

An antimicrobial susceptibility test of isolated K. pneumoniae isolates from the different samples were performed on Muller Hinton agar (Himedia, India) by Kirby-Bauer disk diffusion technique as recommended by Clinical and Laboratory Standards Institute (CLSI) (15). We used thirteen different commonly used antibiotics including; Ciprofloxacin $(5 \mu \mathrm{g})$, Levofloxacin $(5 \mu \mathrm{g})$, Cefotaxime $(30 \mu \mathrm{g})$, Ceftazidime $(30 \mu \mathrm{g})$, Imipenem $(10 \mu \mathrm{g})$, Meropenem $(10 \mu \mathrm{g})$, Piperacillin/Tazobactam $(100 / 10 \mu \mathrm{g})$, Tetracyclin $(30 \mu \mathrm{g})$, Tobramycin $(10 \mu \mathrm{g})$, Gentamycin(10 $\mu \mathrm{g})$, Chloramphenicol $(30 \mu \mathrm{g})$, Co-trimoxazole $(25 \mu \mathrm{g})$, Nitrofurantoin $(300 \mu \mathrm{g})$. 


\section{Detection of Multidrug resistance (MDR) isolates;}

All the K. pneumoniae isolates, which were non-susceptibility to at least one agent in three or more antimicrobial categories were defined as Multidrug resistance (MDR) isolates (16).

\section{Detection of Beta-lactamase;}

\section{Detection of ESBL producing isolates;}

The initial screening test for the production of ESBL was performed by using Ceftazidime $(30 \mu \mathrm{g})$ and Cefotaxime $(30 \mu \mathrm{g})$ discs (HiMedia, India) by Kirby-Bauer disk diffusion method. A combination disc method was performed to confirm the production of ESBL using Ceftazidime $(30 \mu \mathrm{g})$ and Ceftazidime/Clavulanic acid $(30 / 10 \mu \mathrm{g})$. An increase in zone diameter by $\geq 5 \mathrm{~mm}$ in the disc containing Ceftazidime-Clavulanic acid in comparison to ceftazidime alone confirmed the presence of ESBL (17).

\section{Detection of Metallo beta-lactamase (MBL) and Klebsiella pneumoniae carbapenemase (KPC);}

The initial screening test for the production of MBL or KPC was performed by using Meropenem $(10 \mu \mathrm{g})$ and Imipenem $(10 \mu \mathrm{g})$ discs (Himedia, India) by the Kirby-Bauer disk diffusion method. To confirm the MBL production inhibition zone of the EDTA+Meropenem and Meropenem alone was compared Increase in zone diameter $\geq 5 \mathrm{~mm}$ in the area containing both EDTA and Meropenem is the evidence of the Metallo beta-lactamase producer (18).

For KPC confirmation, two Meropenem (MRP) discs are placed $20 \mathrm{~mm}$ away from the center, one with $20 \mu \mathrm{l}$ of $400 \mu \mathrm{g} / \mathrm{ml}$ PBA and another MRP alone. The inhibition zone of the PBA+ Meropenem and Meropenem alone was compared. The zone of inhibition of MRP+PBA is $\geq 5 \mathrm{~mm}$ that of MRP alone is considered KPC production (18). 


\section{Detection of virulence factors:}

\section{Detection of Hypermucoviscosity;}

The string test where a standard bacteriological loop was used to stretch a mucoviscous string from the bacterial colony was utilized to determine the mucoid phenotype. The formation of a viscous string $>10 \mathrm{~mm}$ was regarded as a positive confirmation of the hypermucoviscous $(\mathrm{Hmv})$ phenotype of the strain (19).

\section{Detection of Biofilm;}

The isolates were subjected to biofilm detection by microtitre plate or tissue culture plate method. Organisms isolated from fresh agar plates were inoculated in $2 \mathrm{ml}$ of Luria Bertani broth (HiMedia, India) with $2 \%$ glucose and incubated at $37^{\circ} \mathrm{C}$ for 24 hours. The culture was then diluted at the ratio of 1:100 with a fresh medium. Each well of sterile 96 well polystyrene tissue culture plates were inoculated with $200 \mu$ l of the diluted culture of different strains isolated from the various sample and incubated at $37^{\circ} \mathrm{C}$ for 24 hours. After incubation, contents of each well were removed by gentle tapping and washed with $0.2 \mathrm{~mL}$ of phosphate buffer saline $(\mathrm{pH} 7.2)$ three times, which remove free-floating bacteria. Biofilm formed by bacteria adherent to the wells were fixed by keeping at $60^{\circ} \mathrm{C}$ for 1 hour and were stained by crystal violet (2\%). Excess stain was removed by using deionized water by rinsing three times and subsequently decolorized with $30 \%$ acetic acid. Optical density (OD) of stained adherent biofilm was obtained by using micro ELISA auto reader at wavelength $570 \mathrm{~nm}$.

Un-inoculated wells containing broth were considered as a negative control. The experiment was performed in triplicate for two times. The average optical density (OD) values of each test strain and negative control were calculated, and final OD values of a test strain were expressed as average OD value of the strain reduced by OD cut-off value (ODc) of the negative control. The interpretation of biofilm production was made according to the criteria of Stepanovic et al. ODc was defined as three standard deviations (SDs) above the mean OD of the negative control (3, 20, 21). 


\section{Serum Bactericidal Activity;}

An inoculum of $25 \mu \mathrm{l}$ (adjusted to $10^{6} \mathrm{CFU} / \mathrm{ml}$ ) prepared from the mid-log phase was diluted by $0.9 \%$ saline and was added to $75 \mu \mathrm{l}$ of pooled human sera contained in a tube. Viable counts (VC) were checked at 1,2 , and $3 \mathrm{~h}$ of incubation at $37^{\circ} \mathrm{C}$. Each strain was tested at least 3 times, and the mean results were expressed as per-cent inoculums. The results were expressed as a percentage of inoculation, and the responses in terms of viable counts were graded from 1 to 6 , as serum sensitive at grades of 1 to 2 , intermediately sensitive at grades of 3 to 4 , and resistant at grades of 5 and $6(22)$.

\section{Statistical analysis:}

Data were analyzed using SPSS version 20.0 (IBM Corp., Armonk, NY, USA). And the comparison of resistance and virulence factors among hypermucoviscous and classical strains were analyzed by performing the Chi-Square test. The significance of differences was evaluated at $P \leq 0.05$.

\section{Results}

\section{Bacterial isolation and identification}

A total of one hundred five non-repetitive pure and microbiologically identified as K. pneumoniae were isolated during the study period from various clinical samples collected in the Department of Microbiology. The majority of K. pneumoniae isolates were isolated from urine (40\%), sputum (30.5\%), wound swabs (16.2\%), Blood (9.5\%), respectively. Hypermucoviscosity was observed in

$29(27.6 \%)$ K. pneumoniae (hmvKP) isolates and remained were $76(72.4 \%)$ classical Klebsiella pneumoniae (cKP) which were determined by string test (Figure 1). 


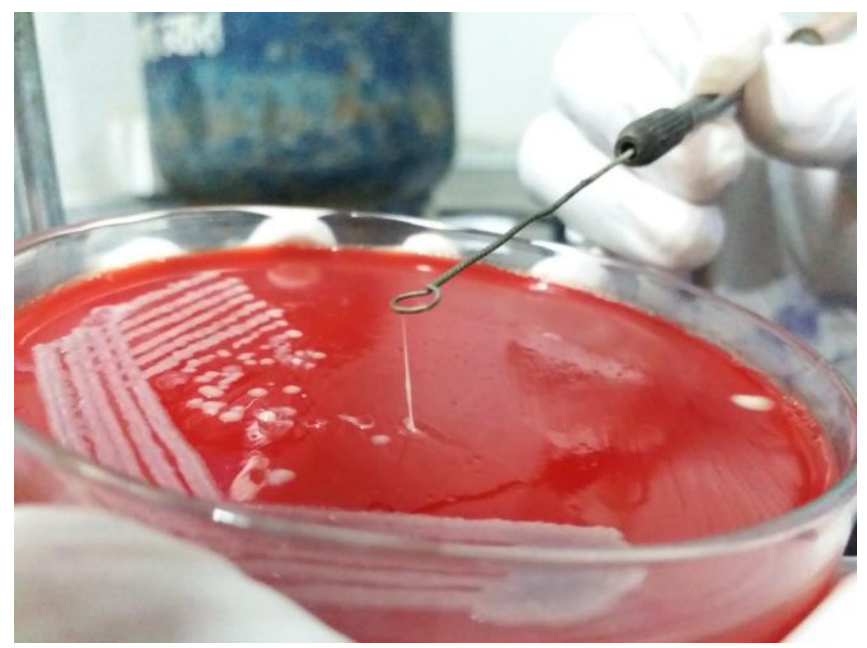

Figure 1: String test positive ( $\geq 10 \mathrm{~mm})$

\section{Antimicrobial Susceptibility Testing}

The antimicrobial susceptibility test of isolated $K$. pneumoniae was determined by the Kirby-Bauer disk diffusion method. Eighty-two isolates (78.1\%) were resistant to cefotaxime, and eighty-four isolates $(80 \%)$ were resistant to ceftazidime, seventy isolates $(66.7 \%)$ were resistant to nitrofurantoin, sixty-six isolates $(62.8 \%)$ were resistant to piperacillin/tazobactam forty-nine isolates (46.7\%) were resistant to both imipenem and meropenem (Table 1).

Table 1: Antibiotics resistance among Hypermucoviscous (hmvKP) and Classical (cKP) strains

\begin{tabular}{|l|c|c|c|c|c|c|}
\hline \multirow{2}{*}{ Antibiotics } & \multicolumn{2}{|c|}{ hmvKP } & \multicolumn{2}{c|}{ cKP } & \multicolumn{2}{c|}{ Total } \\
\cline { 2 - 7 } & $\mathrm{N}$ & $\%$ & $\mathrm{~N}$ & $\%$ & $\mathrm{~N}$ & $\%$ \\
\hline Gentamycin & 12 & 25.5 & 35 & 74.5 & 47 & 44.7 \\
\hline Ciprofloxacin & 14 & 24.1 & 44 & 75.9 & 58 & 55.2 \\
\hline Cefotaxime & 25 & 30.5 & 57 & 69.5 & 82 & 78.0 \\
\hline Ceftazidime & 25 & 29.8 & 59 & 70.2 & 84 & 80 \\
\hline Tetracycline & 7 & 18.4 & 31 & 81.6 & 38 & 36.2 \\
\hline Nitrofurantoin & 17 & 24.3 & 53 & 75.7 & 70 & 66.7 \\
\hline Tobramycin & 12 & 26.1 & 34 & 73.9 & 46 & 43.8 \\
\hline Chloramphenicol & 6 & 16.7 & 30 & 83.3 & 36 & 34.2 \\
\hline Levofloxacin & 11 & 23.4 & 36 & 76.6 & 47 & 44.8 \\
\hline Imipenem & 15 & 30.6 & 34 & 69.4 & 49 & 46.7 \\
\hline Meropenem & 15 & 30.6 & 34 & 69.4 & 49 & 46.7 \\
\hline Piperacillin/Tazobactam & 20 & 30.3 & 46 & 69.7 & 66 & 62.8 \\
\hline Cotrimoxazole & 12 & 21.1 & 45 & 78.9 & 57 & 54.2 \\
\hline
\end{tabular}

'N' = Number of isolates 
A comparison of MDR isolates between hypermucoviscous and classical strains showed the majority of classical strains were found to be multidrug-resistant than hypermucoviscous strains and which was found significantly higher than that of hypermucoviscous strains $(\mathbf{P}<\mathbf{0 . 0 3 8})($ Table 2).

\section{Detection of Beta-lactamase:}

Among the total $105 \mathrm{~K}$. pneumoniae isolates, $53.3 \%$ of the isolates were extended-spectrum beta-lactamase (ESBLs) producers by the phenotypic Combination disk test method. Moreover, when the comparison between classical and hypermucoviscous strains, the rate of ESBL production was found significantly higher among hypermucoviscous isolates than classical strains $(\boldsymbol{P}<\mathbf{0 . 0 0 1 )}$ (Table 2).

Likewise, a total of $28.5 \%$ of $K$. pneumoniae isolates were found to be Metallo-beta lactamase (MBL) producers by the combination disk test method. Similarly, $17.1 \%$ of $K$. pneumoniae isolates were found to be Klebsiella pneumoniae carbapenemase (KPC) producers detected phenotypically by a combination disk test. However, the rate of production of MBLs and KPCs was significantly higher among the hypermucoviscous isolates than classical strains $(\boldsymbol{P}<\mathbf{0 . 0 2 3}$ and $\boldsymbol{P}<\mathbf{0 . 0 2 0}$, respectively) (Table 2).

Table 2: Comparison of MDR and $\beta$-lactamase among hmvKP and cKP

\begin{tabular}{|l|l|l|l|l|}
\hline Tests & & hmvKP $(\mathrm{N})$ & cKP $(\mathrm{N})$ & P-value \\
\hline \multirow{4}{*}{ MDR } & MDR & 15 & 56 & \\
\cline { 2 - 4 } & Non-MDR & 14 & 20 & \multirow{2}{*}{0.038} \\
\hline \multirow{4}{*}{ ESBL } & Positive & 23 & 33 & \multirow{3}{*}{0.001} \\
\cline { 2 - 4 } & Negative & 6 & 43 & \multirow{3}{*}{0.023} \\
\hline \multirow{3}{*}{ MBL } & Positive & 13 & 17 & \\
\cline { 2 - 4 } & Negative & 16 & 59 & 0.020 \\
\hline \multirow{3}{*}{ KPC } & Positive & 9 & 9 & \\
\cline { 2 - 5 } & Negative & 20 & 67 & \\
\hline
\end{tabular}

'N' = Number of isolates 
'hmvKP' = Hypermucoviscous Klebsiella pneumoniae

'cKP' = Classical Klebsiella pneumoniae

\section{Detection of virulence factors:}

Biofilm formations of all isolates were tested by quantitative microtitre plate method. Biofilm production was classified as biofilm producers and non-producers and was compared among hypermucoviscous and classical strains. A total of $24.7 \%(26 / 105)$ K. pneumoniae were biofilm producers detected by the microtitre plate method. Among the total hypermucoviscous strains, $41 \%$ of isolates were biofilm producers, whereas among the total classical strains, it was only $18 \%$ and which was found significantly lower than that of hypermucoviscous strains $(P<0.015)$ (Table 3).

Similarly, among the total isolates, $39 \%(41 / 105)$ of the tested $K$. pneumoniae were found to be serum resistance. However, among hypermucoviscous strains, they showed a significantly higher serum resistance than that among classical strains $(\boldsymbol{P}<\mathbf{0 . 0 1 1})($ Table 3$)$.

Table 3: Comparison of biofilm and serum resistance among hmvKP and cKP

\begin{tabular}{|c|c|c|c|c|}
\hline Tests & & $\operatorname{hmvKP}(\mathrm{N})$ & $\operatorname{cKP}(\mathrm{N})$ & $p$-value \\
\hline \multirow[b]{2}{*}{ Biofilm } & Producers & 12 & 14 & \multirow[b]{2}{*}{0.015} \\
\hline & Non-producers & 17 & 62 & \\
\hline \multirow{2}{*}{$\begin{array}{l}\text { Serum } \\
\text { Killing } \\
\text { Assay }\end{array}$} & Susceptible & 12 & 52 & \multirow[b]{2}{*}{0.011} \\
\hline & Resistance & 17 & 24 & \\
\hline $\mathrm{N}^{\prime}=\mathrm{Nur}$ & olates & & & \\
\hline
\end{tabular}




\section{Discussion}

K. pneumoniae is a common pathogen associated with community and healthcare-associated infections, including respiratory tract infections, urinary tract infections, wounds, and bloodstream infections (23). Klebsiella pathogenicity is due to their virulence factors and their ability to acquire multiple antibiotic resistances $(24,25)$. K. pneumoniae is the most common bacteria after $E$. coli, among the Enterobacteriaceae family causing invasive infections and those invasive infections by K. pneumoniae has been associated with comorbidities conditions like cancer and diabetes (26).

A laboratory-based cross-sectional study was conducted from February 2018 to July 2018 among patients visiting Manmohan Memorial Teaching Hospital, Swoyambhu, Kathmandu, Nepal. A total of $105 \mathrm{~K}$. pneumoniae isolates were collected from different clinical samples. A hypermucoviscous $K$. pneumoniae strain was defined based on a positive string test. Hypermucoviscous $K$. pneumoniae, namely hmvKP are more invasive and associated with severe infections that can be monitored in routine practice by string test (27-29). To our knowledge, this is the first study focused on hypermucoviscous K. pneumoniae from Nepal. In our research, $29(27.6 \%)$ hypermucoviscous strains of $K$. pneumoniae were identified by a positive string test. This percentage is lower than that reported from other Asian countries Taiwan $(80 \%)$, China (45.7\%), South Korea (78\%) (30) respectively. It was showing that the hypermucoviscous strain of $K$. pneumoniae is present in our hospital settings. As they can cause various invasive severe infections; they are a real threat to our health.

$K$ pneumoniae isolates like other Enterobacteriaceae are increasingly resistant to multiple antimicrobial agents, including aminoglycosides, quinolones, and the third-generation cephalosporins (31). Among the total 105 K. pneumoniae, higher resistance was observed in ceftazidime $(80 \%)$, followed by cefotaxime $(78 \%)$, nitrofurantoin $(66.7 \%)$, piperacillin-tazobactam (62.8\%), ciprofloxacin $(55.2 \%)$, cotrimoxazole $(54.2 \%)$, imipenem $(46.7 \%)$ and meropenem (46.7\%). In the study carried out by Parajuli NP et al. in 2017, all isolates of K. pneumoniae were resistant to cotrimoxazole and cefotaxime, $86.4 \%$ to ciprofloxacin, $81.0 \%$ to piperacillintazobactam, $48.6 \%$ of isolates resistant to both imipenem and meropenem (32). Developing 
countries like Nepal, where the health care delivery system is particularly poor, and also prior use of antibiotics like carbapenems without its susceptibility testing might be the cause for the increasing resistance towards the option drugs like carbapenems. In the study by Gharrah et al. in $2017,49 \%$ of isolates were resistant to cefotaxime, and $40 \%$ were resistant to ceftazidime (28). Similarly, in the study by Li and Sun et al. in 2014, out of 88 K. pneumoniae isolates highest resistance was observed in chloramphenicol (42\%), followed by tetracycline (39.7\%), cefotaxime (37.5\%), ciprofloxacin (25.0\%), ceftazidime (23.8\%) and piperacillin-tazobactam (22.7\%) (33). Multidrug resistance (MDR) is defined as acquired non-susceptibility to at least one agent in three or more antimicrobial categories (16). In the study by Nepal et al. in $2017,59 \%$ of $K$. pneumoniae were Multidrug resistance isolates (34). However, our study showed $67.6 \%(71 / 105)$ of $K$. pneumoniae were Multidrug resistance. This shows that the Multidrug resistance isolates are increasing yearly in Nepal. These comparisons suggested that developing countries are facing more problems of antimicrobial resistance, which might be the cause of their easy availability, and lack of guidelines for antibiotics use could be the reason for increasing MDR K. pneumoniae. Therefore, an appropriate standard guideline for antibiotics use should be adopted before the use of antibiotics, and antibiotics like carbapenems are only be used after their susceptibility testing.

Beta-lactams are the choice of drug in the infections of $K$. pneumoniae, but bacterial resistance to beta-lactam antibiotics is increasing globally, causing significant morbidity and mortality (36). Extended-spectrum beta-lactamase(ESBL) production is substantially increasing and is now recognized as a worldwide problem (37). The proportion of $K$. pneumoniae producing ESBL was variable among the various countries, $12 \%$ in the United States, 33\% in Europe, $52 \%$ in Latin America, and $28 \%$ in Western Pacific (38). In the study of Mustafa and Rasha et al. in $2017,50 \%$ of $K$. pneumoniae isolates were ESBLs producer (28). A similar result was found in our study, where $53.5 \%$ of $K$. pneumoniae isolates were ESBL producers. Carbapenems are the preferred antibiotics for the treatment of infections caused by ESBL K. pneumoniae. However, carbapenem resistance in $K$. pneumoniae attributed to the production of carbapenemases like Metallo betalactamase (such as IMP, VIM, and NDM) and production of $K$. pneumoniae carbapenemase 
(KPC), causing the severe clinical problems and complicating the treatment (39-41). In our study, $46.7 \%(49 / 105)$ of $K$. pneumoniae were found to be resistant to carbapenems (Imipenem and Meropenem) by the disk diffusion method. However, $28.7 \%$ (30/105) of $K$. pneumoniae were MBL producers and were detected by phenotypic combination disk test method. This result is higher than that reported by Nepal et al 2017 , where $12.8 \%$ of $K$. pneumoniae were MBL producers (34). Similarly, $17.1 \%(18 / 105)$ of $K$. pneumoniae isolates were found to be KPC producers in our study. This result is found to be higher than the result reported by Parajuli et al in 2017, where $10.8 \%$ of $K$. pneumoniae were KPC producers (32). The increase in the rate of carbapenem resistance might be due to common use of carbapanems during the preliminary treatments use without their susceptibility testing.

In addition to the production of carbapenemase, the microbial biofilm formation, and serum resistance contribute these isolate to become more virulent and make difficulties in their management $(39,41,42)$. Microbial biofilm formation and development have been reported to be a significant pathogenic factor of $K$. pneumoniae infections, as biofilm protects bacteria from exposure to the antimicrobials (43). In our study, $24.7 \%$ of $K$. pneumoniae were biofilm producers, which are less than that reported by Vuotto and Longo et al. in 2017 , where $49.2 \%$ of K. pneumoniae were biofilm producers (35). Similarly, Serum resistance in the different bacterial systems has been critical for their survival and establishments of disease, several mutations in bacteria resulting in loss of serum resistance, making several bacterial pathogens avirulent (44). Serum resistance is one of the major pathogenicity of $K$. pneumoniae. In our study, $39 \%$ of isolates were resistant to serum bactericidal activity, which is similar to that reported by Mustafa and Rasha et al., where $40.0 \%$ of isolates were resistant to serum bactericidal activity (28).

Although in our study, we found the expression of higher virulence factors and antibiotic resistance among the hypermucoviscous strains than classical strains, this result needed to be confirmed by the other confirmatory molecular techniques. Due to limited resources, here in our study, we were unable to confirm this result by molecular methods, and only phenotypic characteristics were studied. Therefore, further their molecular study required before the conclusion. 


\section{Conclusion}

Phenotypic comparison between hypermucoviscous and classical strains in our study, we found both the expression of virulence factors and antimicrobial resistance was significantly higher among the hypermucoviscous strains. While being the first study on hypermucoviscous Klebsiella pneumoniae their prevalence in our hospital settings may increase the risk of morbidity and mortality. Hence, timely diagnosis and appropriate treatment strategies are crucial to reduce the dissemination of hypermucoviscous strains.

\section{Acknowledgements}

I would like to express the deepest gratitude and cordial appreciation to Prof Dr. Basista Prasad Rijal, Dr. Deliya Poudel, Mr. Sheshkant Poudyal, Mr. Santosh Khanal and all the staffs of Manmohan Memorial Teaching Hospital and Manmohan memorial institute of health sciences for all the help rendered while carrying out this research. I thank the entire participants without whom this study would not have been possible.

\section{Abbreviations}

hmvKP- Hypermucoviscous Klebsiella pneumoniae

cKP- Classical Klebsiella pneumoniae

MDR- Multidrug resistance,

ESBL- Extended Spectrum Beta-lactamase

MBL-Metallo Beta-lactamase

KPC- Klebsiella pneumoniae carbapenemase

\section{Funding}

This research did not receive any specific grant from funding agencies in the public, commercial, or not-for-profit sectors 


\section{Consent}

Written informed consent was obtained from the patients for publication of this research article. A copy of the written consent is available for review by the Editor-in-Chief of this journals on request.

\section{Competing interests}

The authors declare that they have no competing interests.

\section{Authors contributions}

Tika Bahadur Thapa wrote the manuscript, performed the statistical analysis and designed the study. Tika Bahadur Thapa and Govardhan Joshi were the principal investigators of the clinical data. Manisha Sapkota, Sujina Maharjan, Nisha Giri, Puspa Raj Khanal and Ojaswee Shrestha contributed in data collection, sample collection and laboratory interventions. All authors read and approved the final manuscript.

\section{References}

1. Bagley ST. Habitat association of Klebsiella species. Infection control: IC. 1985;6(2):52-8. 2. Rock C, Thom KA, Masnick M, Johnson JK, Harris AD, Morgan DJ. Frequency of Klebsiella pneumoniae carbapenemase (KPC)-producing and non-KPC-producing Klebsiella species contamination of healthcare workers and the environment. Infection control and hospital epidemiology. 2014;35(4):426-9.

3. Biofilm protocol optimization for Pseudomonas aeruginosa. ImQuest Bio Sciences.

4. Dao TT, Liebenthal D, Tran TK, Ngoc Thi Vu B, Ngoc Thi Nguyen D, Thi Tran HK, et al. Klebsiella pneumoniae oropharyngeal carriage in rural and urban Vietnam and the effect of alcohol consumption. PLoS One. 2014;9(3):e91999.

5. $\quad$ Tsay RW, Siu LK, Fung CP, Chang FY. Characteristics of bacteremia between community-acquired and nosocomial Klebsiella pneumoniae infection: risk factor for mortality and the impact of capsular serotypes as a herald for community-acquired infection. Arch Intern Med. 2002;162(9):1021-7.

6. Meatherall BL, Gregson D, Ross T, Pitout JD, Laupland KB. Incidence, risk factors, and outcomes of Klebsiella pneumoniae bacteremia. The American Journal of medicine. 2009;122(9):866-73.

7. Korvick JA, Hackett AK, Yu VL, Muder RR. Klebsiella pneumonia in the modern era: clinicoradiographic correlations. Southern medical Journal. 1991;84(2):200-4.

8. Lee CR, Lee JH, Park KS, Jeon JH, Kim YB, Cha CJ, et al. Antimicrobial Resistance of Hypervirulent Klebsiella pneumoniae: Epidemiology, Hypervirulence-Associated Determinants, and Resistance Mechanisms. Frontiers in cellular and infection microbiology. 2017;7:483. 
9. Cheng DL, Liu YC, Yen MY, Liu CY, Wang RS. Septic metastatic lesions of pyogenic liver abscess. Their association with Klebsiella pneumoniae bacteremia in diabetic patients. Arch Intern Med. 1991;151(8):1557-9.

10. Liu YC, Cheng DL, Lin CL. Klebsiella pneumoniae liver abscess associated with septic endophthalmitis. Arch Intern Med. 1986;146(10):1913-6.

11. Boucher HW, Talbot GH, Bradley JS, Edwards JE, Gilbert D, Rice LB, et al. Bad bugs, no drugs: no ESKAPE! An update from the Infectious Diseases Society of America. Clinical infectious diseases : an official publication of the Infectious Diseases Society of America. 2009;48(1):1-12.

12. Kuehn BM. "Nightmare" bacteria on the rise in US hospitals, long-term care facilities. Jama. 2013;309(15):1573-4.

13. Chung PY. The emerging problems of Klebsiella pneumoniae infections: carbapenem resistance and biofilm formation. FEMS microbiology letters. 2016;363(20).

14. Paczosa MK, Mecsas J. Klebsiella pneumoniae: Going on the Offense with a Strong Defense. Microbiology and molecular biology reviews : MMBR. 2016;80(3):629-61.

15. CLSI. Performance Standards for Antimicrobial Disk

Susceptibility Tests; Approved Standard-

Twelfth Edition. 2015.

16. Magiorakos AP, Srinivasan A, Carey RB, Carmeli Y, Falagas ME, Giske CG, et al. Multidrug-resistant, extensively drug-resistant and pandrug-resistant bacteria: an international expert proposal for interim standard definitions for acquired resistance. Clin Microbiol Infect. 2012;18(3):268-81.

17. Rawat D, Nair D. Extended-spectrum beta-lactamases in Gram Negative Bacteria. Journal of global infectious diseases. 2010;2(3):263-74.

18. Tsakris A, Poulou A, Pournaras S, Voulgari E, Vrioni G, Themeli-Digalaki K, et al. A simple phenotypic method for the differentiation of metallo- $\beta$-lactamases and class A KPC carbapenemases in Enterobacteriaceae clinical isolates. Journal of antimicrobial chemotherapy. 2010;65(8):1664-71.

19. Lee HC, Chuang YC, Yu WL, Lee NY, Chang CM, Ko NY, et al. Clinical implications of hypermucoviscosity phenotype in Klebsiella pneumoniae isolates: association with invasive syndrome in patients with community-acquired bacteraemia. Journal of internal medicine. 2006;259(6):606-14.

20. Hassan A, Usman J, Kaleem F, Omair M, Khalid A, Iqbal M. Evaluation of different detection methods of biofilm formation in the clinical isolates. Brazilian Journal of Infectious Diseases. 2011;15(4):305-11.

21. Mishra SK, Basukala P, Basukala O, Parajuli K, Pokhrel BM, Rijal BP. Detection of biofilm production and antibiotic resistance pattern in clinical isolates from indwelling medical devices. Current microbiology. 2015;70(1):128-34.

22. Sahly H, Aucken H, Benedi VJ, Forestier C, Fussing V, Hansen DS, et al. Increased serum resistance in Klebsiella pneumoniae strains producing extended-spectrum betalactamases. Antimicrobial agents and chemotherapy. 2004;48(9):3477-82.

23. Bachman MA, Oyler JE, Burns SH, Caza M, Lépine F, Dozois CM, et al. <span class="named-content genus-species" id="named-content-1">Klebsiella pneumoniae $</$ span $>$ Yersiniabactin Promotes Respiratory Tract Infection through Evasion of Lipocalin 2. Infection and Immunity. 2011;79(8):3309-16.

24. Gharrah MM, Mostafa El-Mahdy A, Barwa RF. Corrigendum to \&\#x201c;Association between Virulence Factors and Extended Spectrum Beta-Lactamase Producing Klebsiella pneumoniae Compared to Nonproducing Isolates\&\#x201d. Interdisciplinary Perspectives on Infectious Diseases. 2018;2018:2.

25. Hazen TH, Zhao L, Boutin MA, Stancil A, Robinson G, Harris AD, et al. Comparative Genomics of an IncA/C Multidrug Resistance Plasmid from Escherichia coli and Klebsiella Isolates from Intensive Care Unit Patients and the Utility of Whole-Genome Sequencing in Health Care Settings. Antimicrobial agents and chemotherapy. 2014;58(8):4814-25.

26. Vading M, Nauclér P, Kalin M, Giske CG. Invasive infection caused by Klebsiella pneumoniae is a disease affecting patients with high comorbidity and associated with high longterm mortality. PLOS ONE. 2018;13(4):e0195258. 
27. Dobrindt U. (Patho-)Genomics of Escherichia coli. International Journal of medical microbiology : IJMM. 2005;295(6-7):357-71.

28. Gharrah MM, Mostafa El-Mahdy A, Barwa RF. Association between Virulence Factors and Extended Spectrum Beta-Lactamase Producing Klebsiella pneumoniae Compared to Nonproducing Isolates. Interdisciplinary Perspectives on Infectious Diseases. 2017;2017:14.

29. Shin J, Ko KS. Comparative Study of Genotype and Virulence in CTX-M-Producing and Non-Extended-Spectrum- $\beta$-Lactamase-Producing <span class="named-content genus-species" id="named-content-1">Klebsiella pneumoniae $</$ span $>$ Isolates. Antimicrobial agents and chemotherapy. 2014;58(4):2463-7.

30. Shon AS, Bajwa RP, Russo TA. Hypervirulent (hypermucoviscous) Klebsiella pneumoniae: a new and dangerous breed. Virulence. 2013;4(2):107-18.

31. Coque TM, Oliver A, Perez-Diaz JC, Baquero F, Canton R. Genes encoding TEM-4, SHV-2, and CTX-M-10 extended-spectrum beta-lactamases are carried by multiple Klebsiella pneumoniae clones in a single hospital (Madrid, 1989 to 2000). Antimicrobial agents and chemotherapy. 2002;46(2):500-10.

32. Parajuli NP, Acharya SP, Mishra SK, Parajuli K, Rijal BP, Pokhrel BM. High burden of antimicrobial resistance among gram negative bacteria causing healthcare associated infections in a critical care unit of Nepal. Antimicrobial resistance and infection control. 2017;6:67.

33. Li W, Sun G, Yu Y, Li N, Chen M, Jin R, et al. Increasing occurrence of antimicrobialresistant hypervirulent (hypermucoviscous) Klebsiella pneumoniae isolates in China. Clinical infectious diseases : an official publication of the Infectious Diseases Society of America. 2014;58(2):225-32.

34. Nepal K, Pant ND, Neupane B, Belbase A, Baidhya R, Shrestha RK, et al. Extended spectrum beta-lactamase and metallo beta-lactamase production among Escherichia coli and Klebsiella pneumoniae isolated from different clinical samples in a tertiary care hospital in Kathmandu, Nepal. Ann Clin Microbiol Antimicrob. 2017;16(1):62.

35. Vuotto C, Longo F, Pascolini C, Donelli G, Balice MP, Libori MF, et al. Biofilm formation and antibiotic resistance in Klebsiella pneumoniae urinary strains. Journal of applied microbiology. 2017;123(4):1003-18.

36. Mimi Atmani S, Messai Y, Alouache S, Fernandez R, Estepa V, Torres C, et al. Virulence characteristics and genetic background of ESBL-producing Klebsiella pneumoniae isolates from wastewater2015. 103-12 p.

37. Paterson DL, Bonomo RA. Extended-Spectrum $\beta$-Lactamases: a Clinical Update. Clinical microbiology reviews. 2005;18(4):657-86.

38. Masterton RG, Turner PJ. Overview of the Meropenem Yearly Susceptibility Test Information Collection (1997-2004). Diagnostic Microbiology and Infectious Disease. 2005;53(4):245-6.

39. Colodner R, Raz R, Chazan B, Sakran W. Susceptibility pattern of extended-spectrum ?lactamase producing bacteria isolated from inpatients to five antimicrobial drugs in a community hospital in Northern Israel2004. 409-10 p.

40. Cagnacci S, Gualco L, Roveta S, Mannelli S, Borgianni L, Docquier JD, et al.

Bloodstream infections caused by multidrug-resistant Klebsiella pneumoniae producing the carbapenem-hydrolyzing VIM-1 metallo-beta-lactamase: first Italian outbreak. The Journal of antimicrobial chemotherapy. 2008;61(2):296-300.

41. Nordmann P, Cuzon G, Naas T. The real threat of Klebsiella pneumoniae carbapenemase-producing bacteria. The Lancet Infectious diseases. 2009;9(4):228-36.

42. Sanchez CJ, Jr., Mende K, Beckius ML, Akers KS, Romano DR, Wenke JC, et al. Biofilm formation by clinical isolates and the implications in chronic infections. BMC infectious diseases. 2013;13:47.

43. Vuotto C, Longo F, Balice M, Donelli G, Varaldo P. Antibiotic Resistance Related to Biofilm Formation in Klebsiella pneumoniae. Pathogens. 2014;3(3):743.

44. Elkins C, Morrow KJ, Olsen B. Serum Resistance in Haemophilus ducreyi Requires Outer Membrane Protein DsrA. Infection and Immunity. 2000;68(3):1608-19. 
